\title{
BMJ Open Influence of lifestyle on the FAIM2 promoter methylation between obese and lean children: a cohort study
}

Lijun Wu, ${ }^{1}$ Xiaoyuan Zhao, ${ }^{1}$ Yue Shen, ${ }^{2}$ Guimin Huang, ${ }^{1}$ Meixian Zhang, ${ }^{1}$
Yinkun Yan, ${ }^{1}$ Dongqing Hou, ${ }^{1}$ Linghui Meng,,${ }^{1}$ Junting Liu, ${ }^{1}$ Hong Cheng, ${ }^{1}$ Jie Mi ${ }^{1}$

To cite: Wu L, Zhao $X$, Shen $Y$, et al. Influence of lifestyle on the FAIM2 promoter methylation between obese and lean children: a cohort study. BMJ Open 2015;5:e007670. doi:10.1136/bmjopen-2015007670

- Prepublication history and additional material is available. To view please visit the journal (http://dx.doi.org/ 10.1136/bmjopen-2015007670).

LW and XZ contributed equally.

Received 14 January 2015 Revised 7 April 2015 Accepted 12 April 2015

\footnotetext{
${ }^{1}$ Department of Epidemiology, Capital Institute of Pediatrics, Beijing, China

${ }^{2}$ National Research Institute for Family Planning, Beijing, China
}

Correspondence to Dr Jie Mi;

Jiemi@vip.163.com

\section{ABSTRACT}

Objective: An obesity-related gene, Fas apoptotic inhibitory molecule 2 (FA/M2), is regulated by nutritional state and the methylation levels of the FAIM2 promoter are significantly associated with obesity. Lifestyle factors, such as sedentary behaviour and physical activity, might modify epigenetic patterns that have been related to obesity. Whether the molecular mechanisms by which FAIM2 affects obesity are involved in lifestyle is unclear. This study investigates the potential differences of the FAIM2 promoter methylation with sedentary behaviour and physical activity in obese and lean children.

Design: Cohort study.

Setting: Institute of Pediatrics in China.

Participants: 59 obese cases and 39 lean controls aged 8-18 years recruited from a cross-sectional survey of children from Beijing in 2013.

Primary and secondary outcome measures: The FAIM2 promoter methylation was quantified using the Sequenom MassARRAY platform. Sedentary behaviour and physical activity were investigated using a questionnaire. The influences of different lifestyles on methylation variations in obese and lean children were examined by multiple linear regression.

Results: The methylation levels at seven $\mathrm{CpG}$ sites of the FAIM2 promoter were significantly associated with sedentary behaviour, especially the methylation levels at site -975 , site -413 , sites -362 and -360 , and sites -353 and $-349(p=0.00004,0.00009,0.0006$ and 0.00005 , respectively). There were significant differences between the methylation levels at four $\mathrm{CpG}$ sites in obese and lean participants with high or moderate physical activity level $<150 \mathrm{~min} /$ week.

Conclusions: This study provides the first evidence that there are significant differences in the associations of the FAIM2 promoter methylation with sedentary behaviour and physical activity between obese and lean children. Our results suggest that lifestyle may possibly be mediating the process of the FAIM2 involved in obesity.

\section{INTRODUCTION}

In recent years, the rising incidence of obesity has been a major threat to public health worldwide. ${ }^{1}$ Obesity most likely results

\section{Strengths and limitations of this study}

- This is the first study to evaluate the methylation levels of the Fas apoptotic inhibitory molecule 2 (FAIM2) promoter and lifestyle factors.

- This study may not provide direct evidence that the expression of FAIM2 influences obesity.

- This study investigated the methylation levels in peripheral blood leucocytes, but not in the hypothalamus or adipocytes.

from the complex interaction between genes and lifestyles such as physical activity, diet and behaviour. ${ }^{2}$ Current evidence indicated that lifestyle factors might modify epigenetic patterns, ${ }^{3}$ and DNA methylation has been suggested to be associated with the development of obesity. ${ }^{4}$

Fas apoptotic inhibitory molecule 2 (FAIM2), a $35.1 \mathrm{kDa}$ membrane protein highly expressed in the hippocampus, regulates Fas ligand-mediated apoptosis in neurons. ${ }^{5}$ The single nucleotide polymorphism (SNP) rs7138803 near FAIM2 was reported to be associated with obesity based on genome-wide association (GWA) studies in Caucasians, ${ }^{6}$ and replicated studies showed that this SNP was significantly associated with obesity in Asian populations. ${ }^{78}$ FAIM2 was regulated by nutritional state ${ }^{9}$ and the methylation levels of the FAIM2 promoter were significantly associated with obesity, ${ }^{10}$ but whether the molecular mechanism by which FAIM2 affects obesity is involved in lifestyle has not been clarified.

Because obesity-related lifestyle factors might modify epigenetic patterns and the methylation levels of the FAIM2 promoter are significantly associated with obesity, we hypothesise that lifestyle factors play a role in the methylation of the FAIM2 promoter. The aim of this study was to investigate the differences of the methylation levels of the FAIM2 promoter between obese and lean participants according to different sedentary behaviour and physical activity. 


\section{METHODS}

Population

The cohort included 59 obese and 39 lean participants randomly recruited from a cross-sectional survey of children from Beijing aged 8-18 years in 2013. The survey was a physical fitness and health surveillance of Beijing school students, and included a questionnaire, medical examination, anthropometric measurement and collected venipuncture blood samples $(n=3143$; boys $50 \%)$. Obesity was diagnosed by the Chinese age-specific and sex-specific body mass index (BMI) cut-offs (see online supplementary table S1) and fat mass percentage (FMP) $>40$. Leanness was diagnosed by WHO BMI cut-offs and FMP $<15$. The research budget limited the sample size in the study. In future studies, we hope to examine the methylation levels of the FAIM2 promoter in greater sample sizes.

We obtained written informed consent from all participants (or their parents/guardians).

\section{Measurement of anthropometric parameters and biochemical analyses}

The anthropometric measurements included FMP, weight and height. All instruments were validated according to the methods of the manufacturers. FMP was measured using a body composition analyser (InBody 720, Biospace Co, Ltd, Seoul, Korea). BMI was calculated as weight in kilograms divided by square of height in metres. Blood pressure was measured via auscultation using a standard clinical sphygmomanometer.

Levels of triglycerides, high-density lipoprotein cholesterol, low-density lipoprotein cholesterol, total cholesterol and fasting plasma glucose were examined using an automatic biochemical analyser (Olympus chemistry analyser AU640, Olympus Optical Co, Ltd, Tokyo, Japan) using commercial kits (Olympus Optical Co, Ltd, Tokyo, Japan).

\section{Measurement of sedentary behaviour and physical activity}

A validated questionnaire was used to investigate sedentary behaviour and physical activity in children. The questionnaire referred to the questionnaire used in the study by Meng et $a l^{11}$ The questionnaires were completed by parents or guardians. The questions were retrospective and the questionnaire collected the data in the past 6 months. Sedentary behaviour was determined by the time spent either watching television or playing video/computer games per day in a week. Moderate physical activity (MPA) was determined by the time spent jogging or playing table tennis, or practising Tai Chi, etc, per week. High physical activity (HPA) was determined by the time spent playing football, basketball or badminton, etc, per week. HPA or MPA level was determined by the time spent on MPA or HPA per week. According to sedentary behaviour and physical activity, we stratified the participants into six groups, including sedentary behaviour $<60 \mathrm{~min} /$ day, sedentary behaviour $\geq 60 \mathrm{~min} /$ day, HPA level $<30 \mathrm{~min} /$ day, HPA level $\geq 30 \mathrm{~min} /$ day, HPA or MPA level $<150 \mathrm{~min} /$ week and HPA or MPA level $\geq 150 \mathrm{~min} /$ week.

\section{DNA methylation analysis}

Genomic DNA was extracted from peripheral white blood cells using a QIAamp DNA Blood Mini Kit (QIAGEN Inc, Valencia, California, USA).

The Sequenom MassARRAY platform (BioMiao Biological Technology, Beijing, China) was used to quantitatively examine the methylation of FAIM2 promoter. The genomic DNA was bisulfite-treated using the EpiTect Bisulfite kit (Qiagen) according to the manufacturer's instructions. The target DNA region was amplified using the bisulfite-modified DNA. The specific primers are listed in online supplementary table S2. The primers were designed using epidesigner (http://www. epidesigner.com), and the methylation ratios were calculated using Epityper software V.1.0 (Sequenom, San Diego, California, USA).

\section{Statistical analysis}

The continuous variables are presented as the mean $\pm \mathrm{SD}$, and the categorical variables are presented as the percentages. Multiple linear regressions were used to investigate the difference in methylation levels between the different groups after adjusting for age and gender, or age, gender and BMI. A logistic regression model was used to investigate the interaction between the methylation levels of the FAIM2 promoter and physical activity regarding the obesity status of the children (the model: obesity $\left.=\mathrm{CpG}+\mathrm{PA}+\mathrm{CpG} \mathrm{G}^{*} \mathrm{PA}\right)$. The $\mathrm{p}$ value $<0.05$ was used to indicate statistically significant differences. We applied multiple testing to correct for multiple comparisons, the false discovery rate (FDR) ${ }^{12}$ approach was used; FDR analysis $(0.05$ as criteria) was applied for six groups (sedentary behaviour $<60 \mathrm{~min} /$ day, sedentary behaviour $\geq 60 \mathrm{~min} /$ day, HPA level $<30 \mathrm{~min} /$ day, HPA level $\geq 30 \mathrm{~min} /$ day, HPA or MPA level $<150 \mathrm{~min} /$ week and HPA or MPA level $\geq 150 \mathrm{~min} /$ week), and $36 \mathrm{CpG}$ sites simultaneously (number of test: $36 \times 6=216$ ). In brief, if the original $p$ value was less than $p$ value for FDR, then it represented statistical significance; otherwise, it suggested non-statistical significance. Data were analysed using SPSS, V.13.0 (SPSS Inc, Chicago, Illinois, USA).

\section{RESULTS}

The characteristics of the study participants are shown in online supplementary table S3. We analysed the associations between the methylation levels of the FAIM2 promoter and the categories of sedentary behaviour or physical activity (see online supplementary tables S4-S6). There was no statistically significant difference after multiple testing. We analysed the associations between the methylation levels of the FAIM2 promoter and sedentary behaviour in the cohort (table 1). The methylation levels at seven CpG sites showed significant differences between obese and lean participants, with 


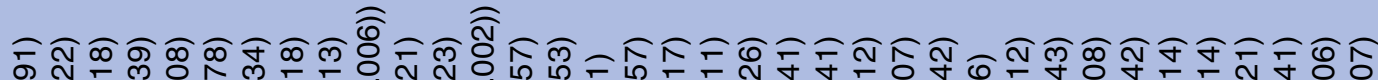

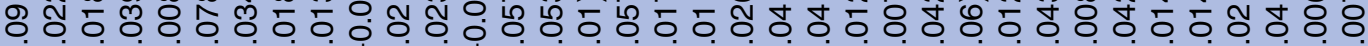

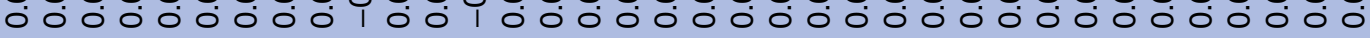
오옹ㅇㅇㅇㅇㅇㅇㅇㅇㅇㅇㅇㅇㅇㅇㅇㅇㅇㅇㅇㅇㅇㅇㅇㅇㅇㅇㅇㅇㅇㅇㅇㅇㅇㅇㅇㅇㅇㅇㅇㅇㅇㅇㅇㅇㅇㅇㅇㅇㅇㅇㅇㅇㅇㅇㅇㅇㅇㅇㅇ

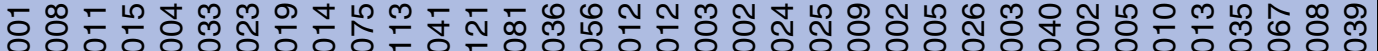

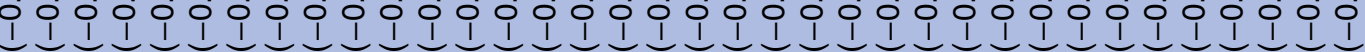
舟

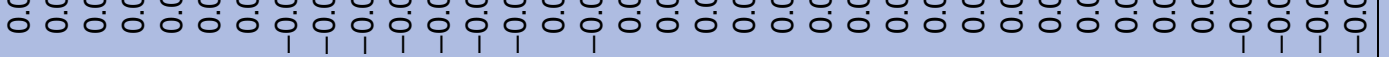

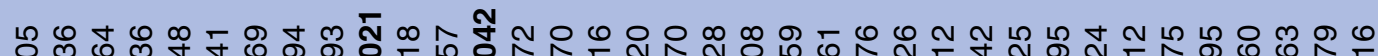

000000000000000000000000000000000000

두 ๒

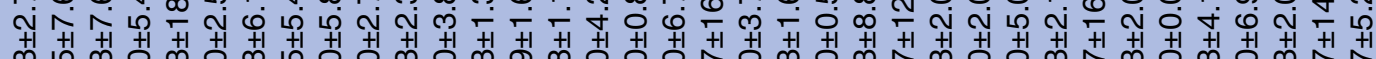
m只

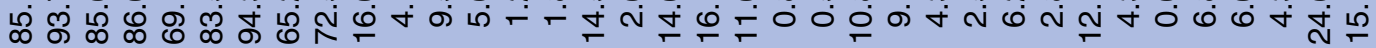

రㄷำ

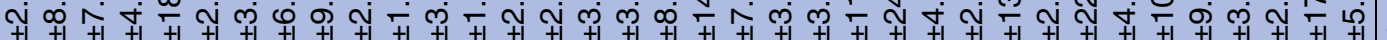

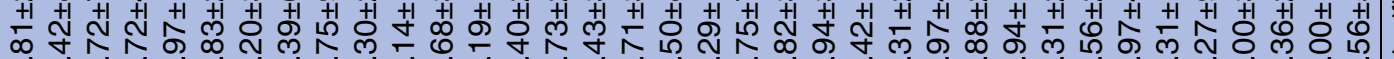

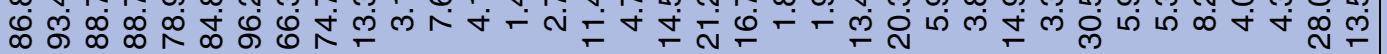

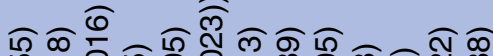

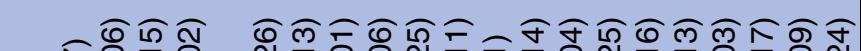

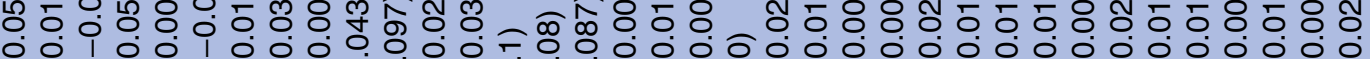

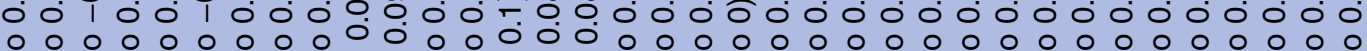

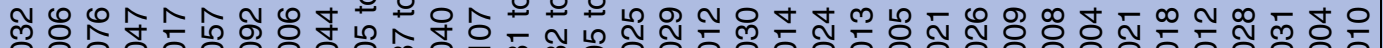

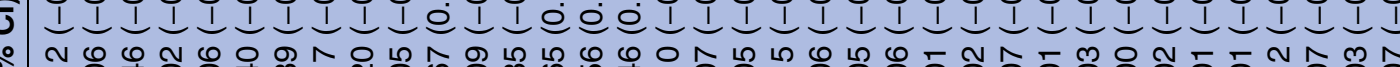

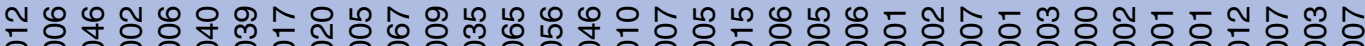

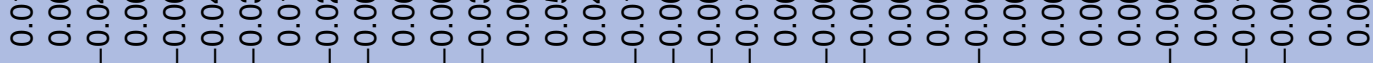

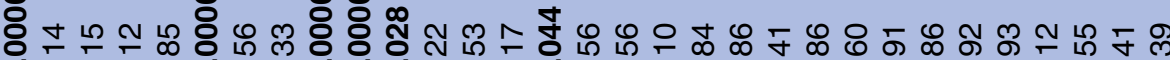
然

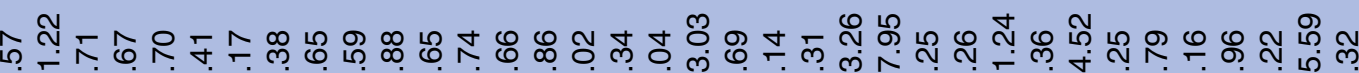
ง

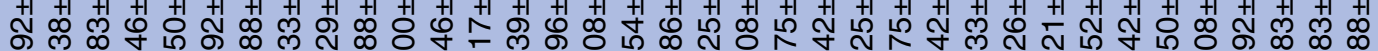

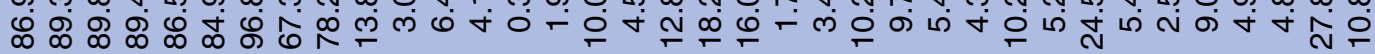

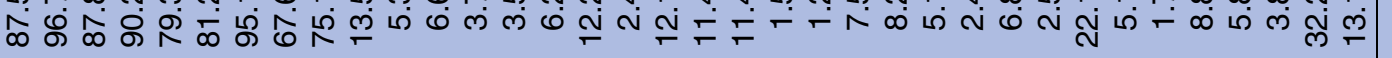

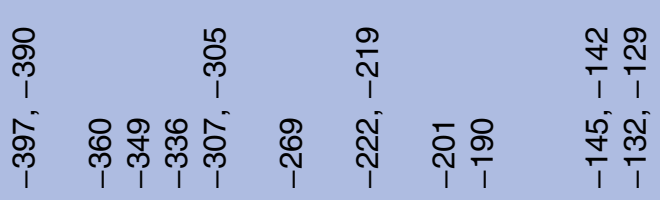

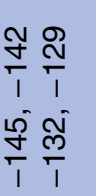




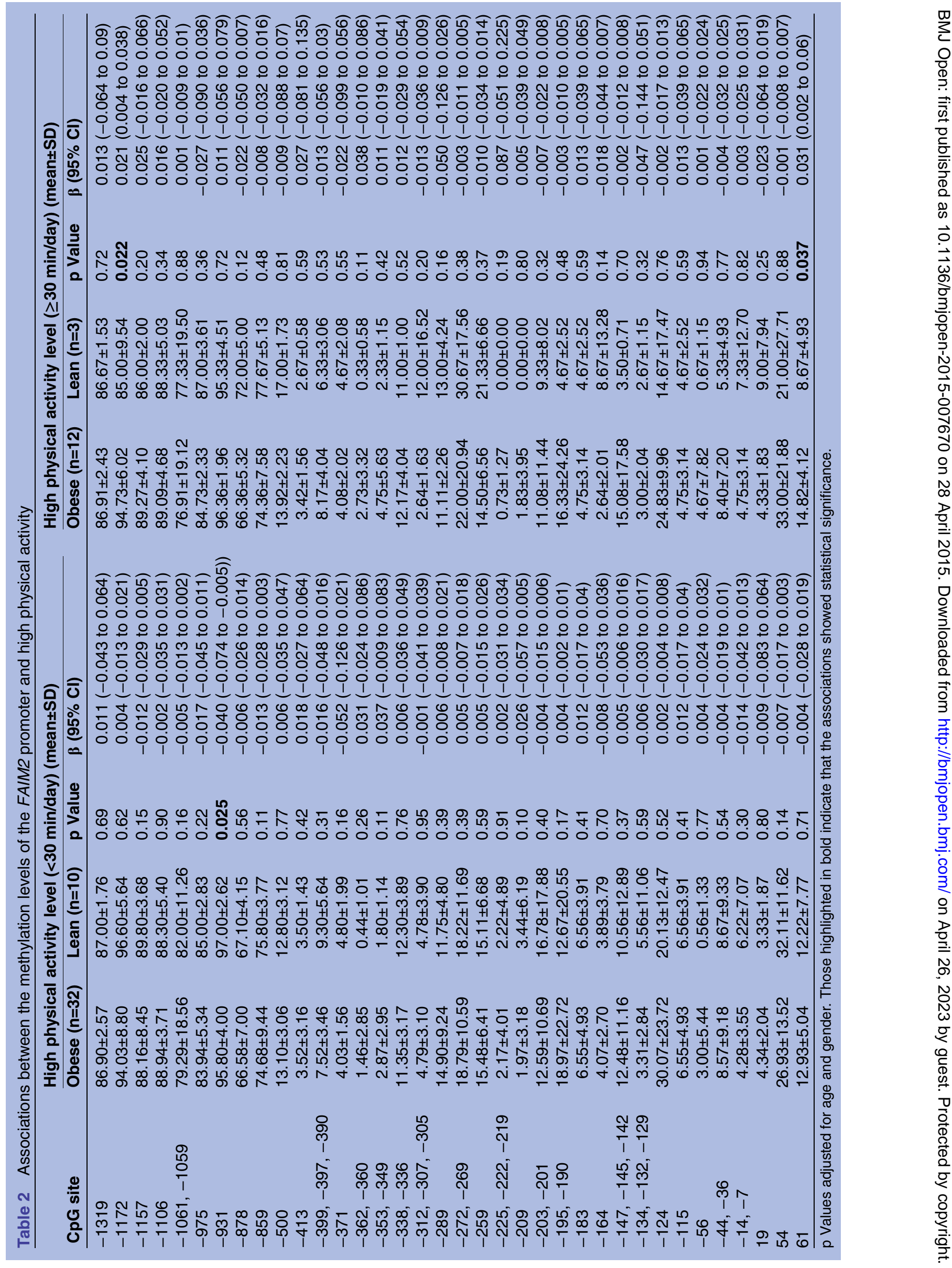


ฟลิ

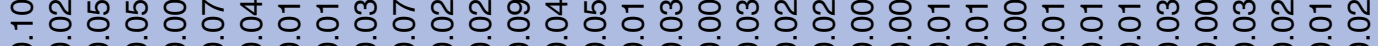
000000000000000000000000000000000000000

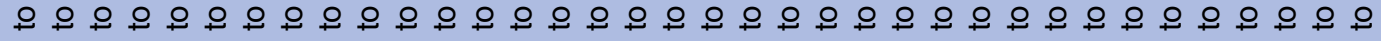

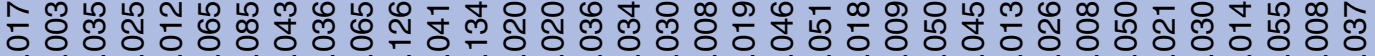
i o i o o o o o o o o o o o o o o o o o o o o o o o o o o o o o o o

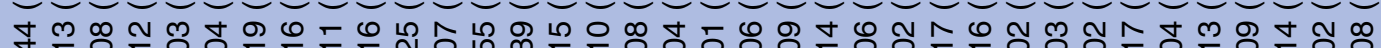

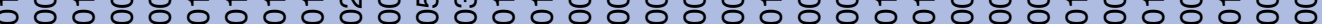

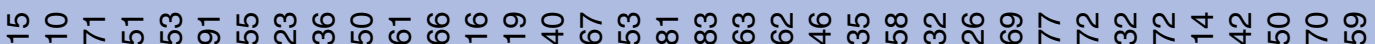

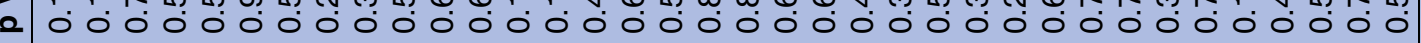

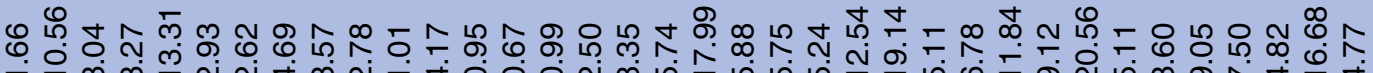

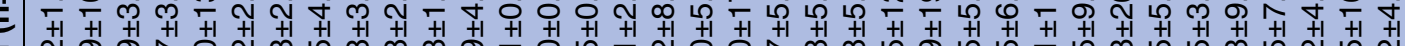

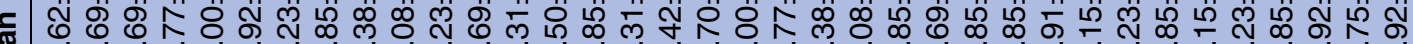
凹

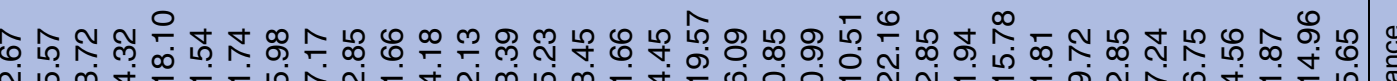
i ग 0 ○ め ठ்

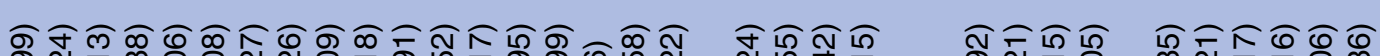

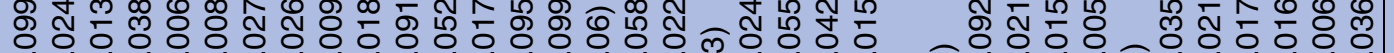

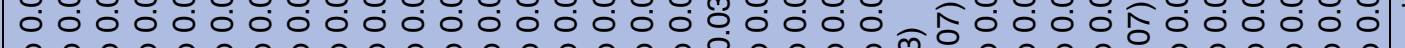

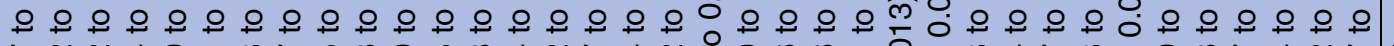
人

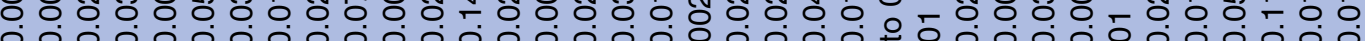

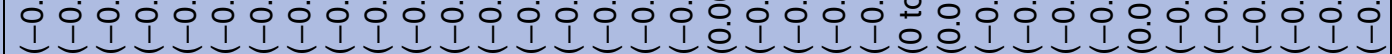

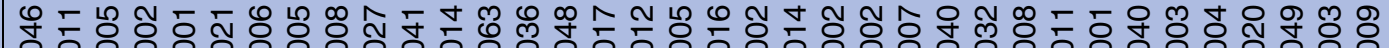

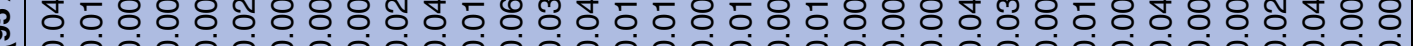

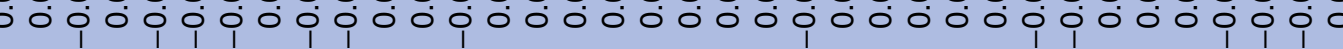

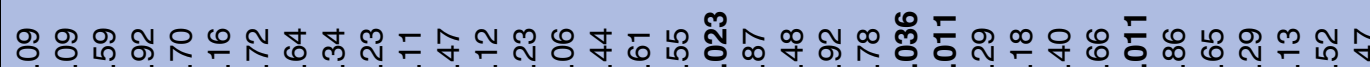

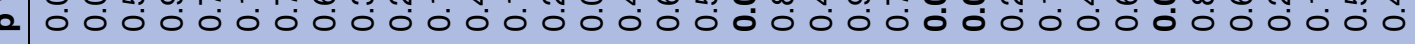

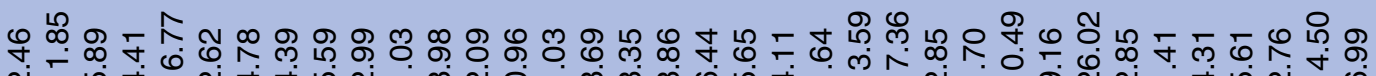
N

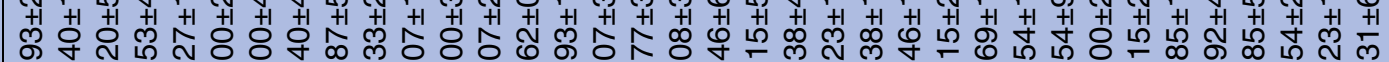
ம் గำ 웅 i ల ల ల్ల

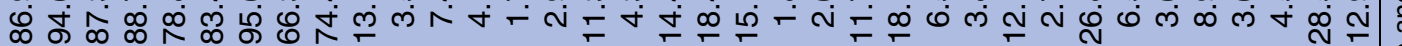

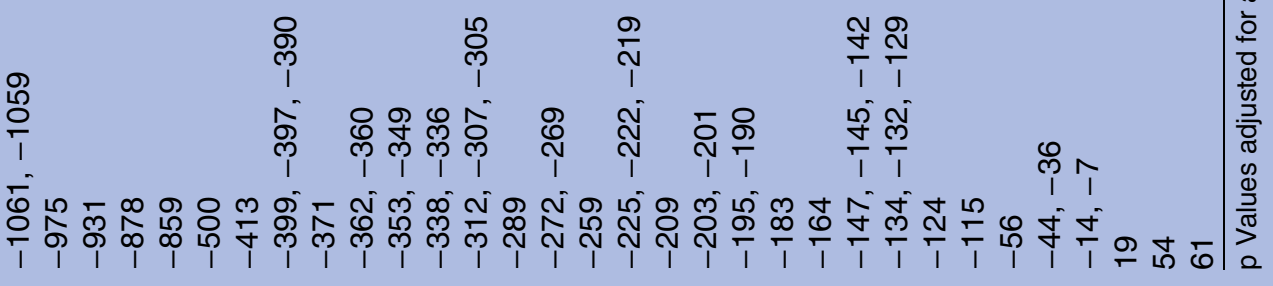


sedentary behaviour $<60 \mathrm{~min} /$ day, especially the methylation levels at site -975 , site -413 , sites -362 and -360 , and sites -353 and $-349(\mathrm{p}=0.00004,0.00009,0.0006$ and 0.00005 , respectively), after adjusting for age and gender. We conducted multiple testing and these four CpG sites reached significance. The methylation levels at two CpG sites (site -500 and site -371 ) showed significant differences between obese and lean participants with sedentary behaviour $\geq 60 \mathrm{~min} /$ day, but these two CpG sites did not reach significance after multiple testing.

Tables 2 and 3 show the associations between the methylation levels of the FAIM2 promoter and physical activity. There were significant differences between the methylation levels at four CpG sites in obese and lean participants with HPA or MPA level $<150 \mathrm{~min} /$ week, but these four CpG sites did not reach significance after multiple testing. There was no statistically significant difference between obese and lean participants with HPA or MPA level $\geq 150 \mathrm{~min} /$ week.

There was no statistically significant interaction between the methylation levels of the FAIM2 promoter and physical activity regarding obesity status after multiple testing (see online supplementary table S7).

\section{DISCUSSION}

Previous studies have shown that lifestyle factors might modify epigenetic patterns ${ }^{3}$ and the methylation levels of the FAIM2 promoter are significantly associated with obesity, ${ }^{10}$ but whether the molecular mechanisms by which FAIM2 affects obesity are involved in lifestyle is unclear. In this study, we investigated the potential associations of the FAIM2 promoter methylation with sedentary behaviour and physical activity in obese and lean children. The methylation levels of the FAIM2 promoter were significantly different in obese and lean children when the groups were stratified by sedentary behaviour and physical activity. There were significant differences in the associations between the FAIM2 promoter methylation with sedentary behaviour and physical activity between obese and lean participants. Our results suggest that lifestyle may possibly be mediating the process of the FAIM2 involved in obesity.

FAIM2 can bind directly to the Fas receptor to protect cells against apoptosis, ${ }^{5}{ }^{13}$ which is a nervous systemspecific inhibitor of Fas/CD95-mediated apoptosis. GWA studies in Caucasians and subsequently replicated studies in Asian populations showed that the SNP rs7138803 near FAIM2 was significantly associated with obesity. ${ }^{6-8}$ Recently, it was reported that the expression of FAIM2 was regulated by high fat feeding in the brain, ${ }^{14}$ and that the methylation levels of the FAIM2 promoter were significantly associated with obesity. ${ }^{10}$

Increasing evidence shows that lifestyle factors may affect epigenetic alterations, especially DNA methylation, which has been identified to be associated with a variety of human diseases including obesity. ${ }^{3}{ }^{4} 15$
For example, physical activity is associated with changes in DNA methylation at many genes related to adipocyte metabolism and type 2 diabetes. ${ }^{16} 17$

This study may not provide direct evidence that the expression of FAIM2 influences obesity, because of the lack of gene expression data from leucocytes and tissues, but our study demonstrated the differences of the associations of the FAIM2 promoter methylation with sedentary behaviour and physical activity between obese and lean children.

There are a few limitations to this study. First, the age range of the obese and lean participants in this study is wide and the sample size is small. Studies with greater sample sizes are needed to examine the associations. Second, obese participants were on average 3.5 years older than lean participants. We want to eliminate the effect by adjusting for age. It is important to adjust the stage of puberty in the analyses, so we will collect data on the stage of puberty in future studies. Third, the data of sedentary behaviour and physical activity were collected by questionnaires. The reliability of questionnaires depends on good communication between the parents and children. Fourth, there were no gene expression data from leucocytes or tissues in the study. Previous studies showed the expression of FAIM2 in the hypothalamus or some other tissues, but there were no expression data of this gene in peripheral blood leucocytes. Fifth, our study investigated the methylation levels in peripheral blood leucocytes, but not in the hypothalamus or adipocytes.

\section{CONCLUSIONS}

In this study we explored, for the first time, the significant differences in the associations between the FAIM2 promoter methylation with sedentary behaviour and physical activity between obese and lean children. Although the function of FAIM2 in the brain remains to be fully researched, identification of methylation changes affected by lifestyle factors will provide important potential to develop new therapies to prevent obesity.

Acknowledgements The authors thank the children and their parents for their participation in this study. They also thank Ping Yang, Zhongjian Su, Xinying Shan, Fangfang Chen and Wenpeng Wang, for their help with sample collection.

Contributors LW designed the study and wrote the manuscript. LW and GH collected the data. YS performed the statistical analysis. XZ, MZ, YY, DH, LM, $\mathrm{JL}$ and $\mathrm{HC}$ collected the DNA samples. All the authors reviewed the manuscript. LW and XZ contributed equally to this work. JM directed the project.

Funding This study was supported by the National Natural Science Foundation of China (grant no. 81200624) and the National Basic Research Program of China (973 Program, grant no. 2013CB530605).

Competing interests None declared.

Patient consent Obtained.

Ethics approval The study was approved by the ethics committees of the Capital Institute of Pediatrics.

Provenance and peer review Not commissioned; externally peer reviewed. 
Data sharing statement No additional data are available.

Open Access This is an Open Access article distributed in accordance with the Creative Commons Attribution Non Commercial (CC BY-NC 4.0) license, which permits others to distribute, remix, adapt, build upon this work noncommercially, and license their derivative works on different terms, provided the original work is properly cited and the use is non-commercial. See: http:// creativecommons.org/licenses/by-nc/4.0/

\section{REFERENCES}

1. Wang Y, Lobstein T. Worldwide trends in childhood overweight and obesity. Int J Pediatr Obes 2006;1:11-25.

2. Xi B, Wang C, Wu L, et al. Influence of physical inactivity on associations between single nucleotide polymorphisms and genetic predisposition to childhood obesity. Am J Epidemiol 2011;173:1256-62.

3. Slattery ML, Curtin K, Sweeney C, et al. Diet and lifestyle factor associations with $\mathrm{CpG}$ island methylator phenotype and BRAF mutations in colon cancer. Int J Cancer 2007;120:656-63.

4. Wang $X$, Zhu H, Snieder $\mathrm{H}$, et al. Obesity related methylation changes in DNA of peripheral blood leukocytes. BMC Med 2010;8:87.

5. Fernandez M, Segura MF, Sole C, et al. Lifeguard/neuronal membrane protein 35 regulates Fas ligand-mediated apoptosis in neurons via microdomain recruitment. $J$ Neurochem 2007;103:190-203.

6. Thorleifsson G, Walters GB, Gudbjartsson DF, et al. Genome-wide association yields new sequence variants at seven loci that associate with measures of obesity. Nat Genet 2009;41:18-24.

7. Wu L, Xi B, Zhang M, et al. Associations of six single nucleotide polymorphisms in obesity-related genes with $\mathrm{BMI}$ and risk of obesity in Chinese children. Diabetes 2010;59:3085-9.
8. Hotta K, Nakamura M, Nakamura T, et al. Association between obesity and polymorphisms in SEC16B, TMEM18, GNPDA2, BDNF, FAIM2 and MC4R in a Japanese population. J Hum Genet 2009:54:727-31.

9. Boender AJ, van Rozen AJ, Adan RA. Nutritional state affects the expression of the obesity-associated genes Etv5, Faim2, Fto, and Negr1. Obesity 2012;20:2420-5.

10. Wu L, Zhao X, Shen Y, et al. Promoter methylation of fas apoptotic inhibitory molecule 2 gene is associated with obesity and dyslipidaemia in Chinese children. Diab Vasc Dis Res 2015;12:217-20.

11. Meng L, Liang Y, Liu J, et al. Prevalence and risk factors of hypertension based on repeated measurements in Chinese children and adolescents. Blood Press 2013;22:59-64.

12. Benjamini $Y$, Hochberg $Y$. Controlling the false discovery rate: a practical and powerful approach to multiple testing. J $R$ Stat Soc $B$ 1995;57:289-300.

13. Somia NV, Schmitt MJ, Vetter DE, et al. LFG: an anti-apoptotic gene that provides protection from Fas-mediated cell death. Proc Natl Acad Sci USA 1999;96:12667-72.

14. Yoganathan $\mathrm{P}$, Karunakaran $\mathrm{S}, \mathrm{Ho} \mathrm{MM}$, et al. Nutritional regulation of genome-wide association obesity genes in a tissue-dependent manner. Nutr Metab (Lond) 2012;9:65

15. Moleres A, Campion J, Milagro FI, et al. Differential DNA methylation patterns between high and low responders to a weight loss intervention in overweight or obese adolescents: the EVASYON study. FASEB J 2013;27:2504-12.

16. Ronn T, Volkov P, Davegardh C, et al. A six months exercise intervention influences the genome-wide DNA methylation pattern in human adipose tissue. PLoS Genet 2013;9:e1003572.

17. Nitert MD, Dayeh T, Volkov $P$, et al. Impact of an exercise intervention on DNA methylation in skeletal muscle from first-degree relatives of patients with type 2 diabetes. Diabetes 2012;61:3322-32. 